\title{
25 Research Square \\ Development a Set of 46 SNP Markers for the Yellow Catfish (Tachysurus Fulvidraco)
}

\section{Huaxing Zhou}

Anhui Academy of Agricultural Sciences https://orcid.org/0000-0002-5569-0083

Tingshuang Pan

Anhui Academy of Agricultural Sciences

\section{Huan Wang}

Anhui Academy of Agricultural Sciences

He Jiang

Anhui Academy of Agricultural Sciences

Jun Ling

Anhui Academy of Agricultural Sciences

\section{Yuting Hu}

Anhui Academy of Agricultural Sciences

Guoqing Duan ( $\sim$ duangq2010@126.com )

Anhui Academy of Agricultural Sciences

\section{Research Article}

Keywords: Yellow catfish, SNP markers, Population genetic analysis, Whole genome resequencing

Posted Date: June 8th, 2021

DOI: https://doi.org/10.21203/rs.3.rs-501513/v1

License: @ (i) This work is licensed under a Creative Commons Attribution 4.0 International License. Read Full License 


\section{Abstract}

The whole genome resequencing was used to develop single nucleotide polymorphisms (SNP) markers for the yellow catfish (Tachysurus fulvidraco). A total of 46 SNP markers were selected from 5550676 genotyping markers which distributed on 26 chromosomes. Of the 46 SNPs analyzed, 35 SNPs conformed to Hardy-Weinberg equilibrium. The observed and expected heterozygosity of these markers ranged from 0.2519 to 0.771 and from 0.265 to 0.5018 , respectively. This set of markers will be of great useful for population genetics of the yellow catfish.

\section{Introduction}

The yellow catfish (Tachysurus fulvidraco), belonging to Bagridae (Nelson et al. 2016), is an important economic species of freshwater fish, widely distributed in China. Due to its delicious taste and high nutritional value, the yellow catfish was favored by the market. With the increasing market demand, this species has suffered overexploitation and declined sharply of its natural stocks over the past few years (Guo et al. 2006; Yu et al. 2019). Therefore, it is essential to understand the population genetics of yellow catfish for the conservation and sustainable exploitation.

Precious genetic studies of this species has been explored using SSR markers (Feng et al. 2009; Hu et al. 2009; Zhang et al. 2009). However, the limitations of SSR markers motived the development of more informative SNP markers for the population genetics of the yellow catfish (Meyer-Sand et al. 2018). Here we characterized a set of 46 SNP markers for the yellow catfish which were analyzed using whole genome resequencing.

\section{Materials And Methods}

The genomic DNA from 129 individuals was sequenced using Illumina HiSeq ${ }^{\text {TM }}$ X Ten platform (Zhou et al. 2021). After reads quality filter and mapping to the reference genome (Gong et al. 2018), 46 SNP markers were randomly selected from 5550676 genotyping markers which distributed on 26 chromosomes. The genetic parameters of these markers, including observed heterozygosity, expected heterozygosity, and the Hardy-Weinberg equilibrium (HWE), were calculated using Popgene v 1.32 (Yeh et al. 1997).

The marker primers were designed by the flanking sequence using Oligo 7 (Rychlik 2007) software and examined for their amplification efficiency. PCR were conducted in $50 \mu \mathrm{L}$ reaction mixtures containing 200 ng template DNA, $5 \mu \mathrm{L} 10 \times$ buffer (TaKaRa, Dalian, China), $4 \mu \mathrm{L} \mathrm{MgCl} 2(2.5 \mathrm{~mol} / \mathrm{L}), 2.5 \mu \mathrm{L}$ dNTP $(2.5 \mathrm{mmol} / \mathrm{L}), 2 \mu \mathrm{L}$ of each primer ( $5 \mu \mathrm{mol} / \mathrm{L})$, and $1 \mathrm{U}$ Taq DNA polymerase $(25 \mathrm{U} / \mu \mathrm{L}$, TaKaRa). PCR conditions were as follows: initial denaturation $\left(95^{\circ} \mathrm{C}, 2 \mathrm{~min}\right)$, then 34 cycles of denaturation $\left(94^{\circ} \mathrm{C}, 40 \mathrm{~s}\right)$, primer annealing $\left(55^{\circ} \mathrm{C}, 50 \mathrm{~s}\right)$, and elongation $\left(72^{\circ} \mathrm{C}, 60\right.$ s) and a final extension $\left(72^{\circ} \mathrm{C}, 10 \mathrm{~min}\right)$.

\section{Results}

All 46 SNP loci were amplified successfully. The observed heterozygosity of these markers varied from 0.2519 to 0.771 , while the expected heterozygosity ranged from 0.265 to 0.5018 (Table 1). A total of 35 loci conformed to Hardy-Weinberg equilibrium $(p>0.01)$ after Bonferroni's correction, insuring the reliability of their application to evaluate larger groups. 
Table 1

Characterization of 46 SNP markers in Tachysurus fulvidraco

\begin{tabular}{|c|c|c|c|c|c|c|c|c|}
\hline SNP ID & $\begin{array}{l}\text { Chr. } \\
\text { NO. }\end{array}$ & Primer sequence $\left(5^{\prime}-3^{\prime}\right)$ & $\begin{array}{l}\text { Size } \\
\text { (bp) }\end{array}$ & $\begin{array}{l}\text { SNP } \\
\text { position } \\
\text { (bp) }\end{array}$ & $\begin{array}{l}\text { SNP } \\
\text { type }\end{array}$ & $H_{0}$ & $H_{e}$ & HWEP \\
\hline \multirow[t]{2}{*}{55823} & \multirow[t]{2}{*}{1} & AGGAGCTTTACAAACACTCAC & \multirow[t]{2}{*}{591} & \multirow[t]{2}{*}{331} & \multirow[t]{2}{*}{$\mathrm{A} / \mathrm{C}$} & \multirow[t]{2}{*}{0.3664} & \multirow[t]{2}{*}{0.3285} & \multirow[t]{2}{*}{ NS } \\
\hline & & CTGTTTGCCTAAAATGCTCCA & & & & & & \\
\hline \multirow[t]{2}{*}{2071} & \multirow[t]{2}{*}{1} & TTTAAATAAGCACAAATAGCAA & \multirow[t]{2}{*}{571} & \multirow[t]{2}{*}{87} & \multirow[t]{2}{*}{$\mathrm{A} / \mathrm{C}$} & \multirow[t]{2}{*}{0.4504} & \multirow[t]{2}{*}{0.3586} & \multirow[t]{2}{*}{ NS } \\
\hline & & AAACTTCCAGAATGTCCCC & & & & & & \\
\hline \multirow[t]{2}{*}{58894} & \multirow[t]{2}{*}{1} & CCAAATAATGAAGCGAAAGCC & \multirow[t]{2}{*}{681} & \multirow[t]{2}{*}{310} & \multirow[t]{2}{*}{$\mathrm{C} / \mathrm{A}$} & \multirow[t]{2}{*}{0.3969} & \multirow[t]{2}{*}{0.4661} & \multirow[t]{2}{*}{ NS } \\
\hline & & ACAATGCAGGAATGATAACCAG & & & & & & \\
\hline \multirow[t]{2}{*}{52924} & \multirow[t]{2}{*}{1} & CTGCCCATGGTCATAATTGGA & \multirow[t]{2}{*}{653} & \multirow[t]{2}{*}{428} & \multirow[t]{2}{*}{$\mathrm{C} / \mathrm{T}$} & \multirow[t]{2}{*}{0.3817} & \multirow[t]{2}{*}{0.4258} & NS \\
\hline & & TGCCACTACCTTATTATCACT & & & & & & \\
\hline 560625 & 2 & AACCGTTAAAATTAGAGATCACAC & 586 & 393 & $\mathrm{G} / \mathrm{T}$ & 0.4198 & 0.3966 & NS \\
\hline & & CAACTTAAGCACAACTCGGTA & & & & & & \\
\hline 599487 & 2 & GAATCTGATCACTGAGCTCGT & 468 & 226 & $\mathrm{C} / \mathrm{T}$ & 0.6870 & 0.4806 & * \\
\hline & & TTTCAAGAACCAGCATGGAC & & & & & & \\
\hline 638351 & 2 & AGTTCTGCCTTCAACACC & 877 & 378 & $\mathrm{~T} / \mathrm{C}$ & 0.2977 & 0.2955 & NS \\
\hline & & TAGTGCCATAGAAAATAGCTT & & & & & & \\
\hline 638434 & 2 & TTCTTACCGTAACTCATGC & 603 & 367 & $\mathrm{G} / \mathrm{T}$ & 0.2519 & 0.3053 & NS \\
\hline & & CACGTCTATGAATCTACTGC & & & & & & \\
\hline 760278 & 3 & CCAACGTGCTATGGTCT & 584 & 278 & $\mathrm{G} / \mathrm{C}$ & 0.4351 & 0.5014 & NS \\
\hline & & ACAAACTGTCTTAAAGGGCTC & & & & & & \\
\hline 938090 & 3 & TCATTTTССТСТTСАTGСАСТ & 565 & 351 & $A / G$ & 0.6947 & 0.4851 & * \\
\hline & & TAGCCAGACATCCCGAA & & & & & & \\
\hline 1149005 & 4 & CAACTCCACAGATGCACT & 364 & 99 & $\mathrm{~A} / \mathrm{T}$ & 0.6183 & 0.4790 & NS \\
\hline & & СААСАТССССТААТТААТССАС & & & & & & \\
\hline 1149027 & 4 & ATTTCAAGCCTTATTGCTC & 694 & 186 & $A / G$ & 0.5802 & 0.4574 & NS \\
\hline & & CTTTTACAACCAAGTGCAT & & & & & & \\
\hline 1487601 & 5 & AAATAGGCTTCATGTGT & 501 & 275 & $\mathrm{~T} / \mathrm{G}$ & 0.6183 & 0.4756 & NS \\
\hline & & TCTTCTTTAACTGGCATC & & & & & & \\
\hline 1798440 & 6 & ACATATACATACGCACACGTT & 498 & 367 & $\mathrm{~T} / \mathrm{C}$ & 0.5420 & 0.4990 & NS \\
\hline
\end{tabular}




\begin{tabular}{|c|c|c|c|c|c|c|c|c|}
\hline SNP ID & $\begin{array}{l}\text { Chr. } \\
\text { NO. }\end{array}$ & Primer sequence $\left(5^{\prime}-3^{\prime}\right)$ & $\begin{array}{l}\text { Size } \\
\text { (bp) }\end{array}$ & $\begin{array}{l}\text { SNP } \\
\text { position } \\
\text { (bp) }\end{array}$ & $\begin{array}{l}\text { SNP } \\
\text { type }\end{array}$ & $H_{0}$ & $H_{e}$ & HWEP \\
\hline & & CCATGTCTGTAATTTTCACGTT & & & & & & \\
\hline \multirow[t]{2}{*}{1855240} & 7 & CAAGCTTATGTTAAAGGCTCT & 570 & 125 & $A / G$ & 0.6641 & 0.4756 & * \\
\hline & & AACTAACGATGTCACACGCTA & & & & & & \\
\hline \multirow[t]{2}{*}{2265537} & 9 & ATCGTCCATGACAAATCACC & 620 & 149 & $\mathrm{C} / \mathrm{T}$ & 0.5420 & 0.3966 & * \\
\hline & & TCTGTTTATTCTCATAGTGCAA & & & & & & \\
\hline \multirow[t]{2}{*}{2477173} & 10 & TAGTGAATAATCATAGGGCTT & 540 & 231 & $\mathrm{G} / \mathrm{T}$ & 0.3206 & 0.2906 & NS \\
\hline & & TATTTCCACAACACGCTCA & & & & & & \\
\hline \multirow[t]{2}{*}{2597747} & 10 & ATCCTAGCCAATGTATTGCTG & 603 & 398 & $\mathrm{C} / \mathrm{T}$ & 0.2977 & 0.2650 & NS \\
\hline & & TGTTAGGTATCGCCACT & & & & & & \\
\hline \multirow[t]{2}{*}{2676641} & 11 & GCCTTTGTTCTTATAGTGCAT & 603 & 337 & $A / G$ & 0.5954 & 0.4836 & NS \\
\hline & & САCTGAGCTCCTGTTACACAC & & & & & & \\
\hline \multirow[t]{2}{*}{2941890} & 12 & TTAGTTGCAGGTTCCTC & 658 & 154 & $\mathrm{~A} / \mathrm{C}$ & 0.5802 & 0.4701 & NS \\
\hline & & AAGTAAGCCTGTATTAGCC & & & & & & \\
\hline \multirow[t]{2}{*}{3171163} & 13 & TTGCCACAAGGTATAACCGAA & 419 & 327 & $\mathrm{~A} / \mathrm{T}$ & 0.4275 & 0.3931 & NS \\
\hline & & GGCTCTAGTATTTTTATCAAACCC & & & & & & \\
\hline \multirow[t]{2}{*}{3171175} & 13 & AATCAGAGCGTTTGAACCAT & 403 & 119 & $T / G$ & 0.6107 & 0.5012 & NS \\
\hline & & САATTCCCTAATTTGCCCTT & & & & & & \\
\hline \multirow[t]{2}{*}{3171187} & 13 & CAGAATCAGTTATGGCCCTC & 693 & 317 & $A / G$ & 0.5725 & 0.5014 & NS \\
\hline & & TTACTTCTGGCTAATTACTGG & & & & & & \\
\hline \multirow[t]{2}{*}{3171263} & 13 & ATCACACTCATTCGGCTT & 558 & 305 & $\mathrm{~T} / \mathrm{C}$ & 0.2672 & 0.3147 & NS \\
\hline & & AAGGCTATCATGCACCCAA & & & & & & \\
\hline \multirow[t]{2}{*}{3171347} & 13 & CAGCCATTAAAATATAGGGAG & 451 & 129 & $A / G$ & 0.6260 & 0.4773 & NS \\
\hline & & CAACACAATTTCGCTGGA & & & & & & \\
\hline \multirow[t]{2}{*}{3319329} & 14 & GCCTCCACCTAGACTCC & 423 & 239 & $A / G$ & 0.5344 & 0.4574 & NS \\
\hline & & AGAATGCGATGTTTACTGCT & & & & & & \\
\hline \multirow[t]{2}{*}{3485859} & 14 & CTAATTGTGCCTTGTATGCAG & 380 & 89 & $\mathrm{G} / \mathrm{T}$ & 0.5649 & 0.4619 & NS \\
\hline & & TGTAAATAATCAGAGCGTGT & & & & & & \\
\hline 3541374 & 15 & ACGTGTACAACTGTTATTACGC & 405 & 233 & $\mathrm{G} / \mathrm{C}$ & 0.5573 & 0.4756 & NS \\
\hline
\end{tabular}




\begin{tabular}{|c|c|c|c|c|c|c|c|c|}
\hline SNP ID & $\begin{array}{l}\text { Chr. } \\
\text { NO. }\end{array}$ & Primer sequence $\left(5^{\prime}-3^{\prime}\right)$ & $\begin{array}{l}\text { Size } \\
\text { (bp) }\end{array}$ & $\begin{array}{l}\text { SNP } \\
\text { position } \\
\text { (bp) }\end{array}$ & $\begin{array}{l}\text { SNP } \\
\text { type }\end{array}$ & $H_{0}$ & $H_{e}$ & HWEP \\
\hline & & GTGTCCGCATCATAAACGAA & & & & & & \\
\hline \multirow[t]{2}{*}{3542145} & 15 & CTAATCTCAGCACATCCG & 450 & 157 & $\mathrm{C} / \mathrm{T}$ & 0.5267 & 0.4228 & NS \\
\hline & & AATCAGATGAAAGGCACAC & & & & & & \\
\hline \multirow[t]{2}{*}{3706234} & 16 & TATTGGCAACAACCAGT & 537 & 372 & $\mathrm{G} / \mathrm{T}$ & 0.5573 & 0.4756 & NS \\
\hline & & GCATAAGACACATAACGCATC & & & & & & \\
\hline \multirow[t]{2}{*}{4029832} & 17 & GTACTGCCAATTTACACAAC & 576 & 385 & $\mathrm{G} / \mathrm{C}$ & 0.6794 & 0.4720 & * \\
\hline & & AAAATTAAAAGCCTTATTAGTCCA & & & & & & \\
\hline \multirow[t]{2}{*}{4030097} & 17 & ACGCTGTTGTCCTACCTG & 426 & 182 & $A / G$ & 0.6336 & 0.4681 & * \\
\hline & & CCCATTTTTCTAGGGGTCCTT & & & & & & \\
\hline \multirow[t]{2}{*}{4057632} & 17 & ATGCTTCATTTCATCCGTTC & 417 & 275 & $\mathrm{C} / \mathrm{T}$ & 0.6794 & 0.5018 & * \\
\hline & & ACCCGCCTTAAATTTGGAC & & & & & & \\
\hline \multirow[t]{2}{*}{4174143} & 18 & GTGACCCGATTTTACATGACC & 470 & 296 & $\mathrm{~A} / \mathrm{T}$ & 0.6183 & 0.4720 & NS \\
\hline & & GCGTTGTGGAATTCTTATCACT & & & & & & \\
\hline \multirow[t]{2}{*}{4329563} & 19 & ССTTCATCTTCTGGATTTTGCG & 561 & 291 & $\mathrm{C} / \mathrm{T}$ & 0.5115 & 0.4102 & NS \\
\hline & & TGACTGCCAACAGATTACCCAA & & & & & & \\
\hline \multirow[t]{2}{*}{4540487} & 20 & CTGTAGGAAAACACCGCTCT & 653 & 330 & $A / G$ & 0.6107 & 0.4701 & NS \\
\hline & & TGCCAATCAACCTACCATGC & & & & & & \\
\hline \multirow[t]{2}{*}{4590446} & 21 & ACAGAGACATCAGTGCAA & 418 & 194 & $\mathrm{~A} / \mathrm{T}$ & 0.6947 & 0.4902 & * \\
\hline & & GACAAAACTCCTTCGGAT & & & & & & \\
\hline \multirow[t]{2}{*}{4931481} & 22 & CCTTAAACACTAGGCTACCAC & 659 & 392 & $\mathrm{C} / \mathrm{T}$ & 0.6260 & 0.4914 & NS \\
\hline & & GAGAAAAGATTTACGCTGCT & & & & & & \\
\hline \multirow[t]{2}{*}{4931542} & 22 & ATAAGTTGCACCAGCGTA & 522 & 105 & $\mathrm{G} / \mathrm{T}$ & 0.5344 & 0.4528 & NS \\
\hline & & GCTGGTCATTTAATAACTAACAC & & & & & & \\
\hline \multirow[t]{2}{*}{4931576} & 22 & TCAGCCGTTACTAGGTT & 657 & 359 & $A / G$ & 0.6947 & 0.4902 & * \\
\hline & & CCACAGACTGACGACCA & & & & & & \\
\hline \multirow[t]{2}{*}{4931603} & 22 & CTGCCTCTATGCCCGAT & 531 & 310 & $\mathrm{G} / \mathrm{T}$ & 0.4885 & 0.4069 & NS \\
\hline & & ААCTGCTGCCTTTGAGACTG & & & & & & \\
\hline 5028968 & 23 & GAAAGCTGCTAGACACA & 584 & 328 & $A / C$ & 0.5344 & 0.4661 & NS \\
\hline
\end{tabular}




\begin{tabular}{|c|c|c|c|c|c|c|c|c|}
\hline SNP ID & $\begin{array}{l}\text { Chr. } \\
\text { NO. }\end{array}$ & Primer sequence $\left(5^{\prime}-3^{\prime}\right)$ & $\begin{array}{l}\text { Size } \\
\text { (bp) }\end{array}$ & $\begin{array}{l}\text { SNP } \\
\text { position } \\
\text { (bp) }\end{array}$ & $\begin{array}{l}\text { SNP } \\
\text { type }\end{array}$ & $H_{0}$ & $H_{e}$ & HWEP \\
\hline & & GTTGGTAATTTTAGCCCTC & & & & & & \\
\hline \multirow[t]{2}{*}{5078926} & 23 & ATGCTAACCACTAAGCCA & 631 & 453 & $\mathrm{C} / \mathrm{T}$ & 0.6031 & 0.4821 & NS \\
\hline & & CCAATTCACATATGCCTT & & & & & & \\
\hline \multirow[t]{2}{*}{5206582} & 24 & ATCTTGGTGACTTTGCGCTT & 539 & 246 & $\mathrm{~A} / \mathrm{G}$ & 0.6641 & 0.4790 & * \\
\hline & & GCGGTTGCCACTATAATCTCA & & & & & & \\
\hline \multirow[t]{2}{*}{5232887} & 25 & GCAAATAAGAAGCACGA & 628 & 308 & $\mathrm{C} / \mathrm{T}$ & 0.5725 & 0.4720 & NS \\
\hline & & CAAGCACCAAGTTAGCTC & & & & & & \\
\hline \multirow[t]{2}{*}{5456365} & 26 & TGGCTTACACCTGATATAGAGGAC & 452 & 177 & $\mathrm{G} / \mathrm{T}$ & 0.7710 & 0.4977 & * \\
\hline & & CAGCGCAAAAGCTTAGCACCA & & & & & & \\
\hline
\end{tabular}

\section{Declarations}

\section{Acknowledgements:}

We would like to thank Huarun Technology Breeding Co. Ltd for the helping of sample collection. This study was funded by the Earmarked Fund of Anhui Fishery Research System (No.2016-84) and Anhui key research and development programs (202004a06020063, 201904f06020002).

\section{Funding}

This study was funded by the Earmarked Fund of Anhui Fishery Research System (No.2016-84) and Anhui key research and development programs (202004a06020063, 201904f06020002).

\section{Conflicts of interest}

The authors declare there are no competing interests.

\section{Availability of data and material}

The datasets analyses during the current study are available in the BioProject Database (Accession no. PRJNA683740).

\section{Authors' contributions}

All authors contributed to the study conception and design. Material preparation, data collection and analysis were performed by Huaxing Zhou, Guoqing Duan, Huan Wang and Tingshuang Pan. The first draft of the manuscript was written by Huaxing Zhou and all authors commented on previous versions of the manuscript. All authors read and approved the final manuscript. 


\section{Ethics approval and consent to participate}

The experimental protocol was established, according to the ethical guidelines of the Basel Declaration and was approved by the Experimental Animal Welfare and Ethical of Anhui Academy of Agricultural Sciences (NO. AAAS 2020-11)

\section{Consent for publication}

Not applicable.

\section{References}

Feng XY, Li ZQ, Xie N, Li JL (2009) Isolation and characterization of twelve novel microsatellites in yellow catfish, Pelteobagrus fulvidraco. Conserv Genet 10: 755-757.

Gong G, Dan C, Xiao S, Guo W, Huang P, Xiong Y, Wu J, He Y, Zhang J, Li X, Chen N, Gui J, Mei J (2018) Chromosomal-level assembly of yellow catfish genome using third-generation DNA sequencing and Hi-C analysis. GigaScience 7: 1-9.

Guo J, Wang Y, Ma H, Yue Y (2006) Microsatellite marker analysis of genetic diversity and phylogenetic relationships in three populations of Pseudobagrus fulvidraco. Amino Acids Biot Resour Sin 28: 5-8.

Hu GF, Liang HW, Li Z, Wang CZ, Wu QC, Liu XJ, Luo XZ, Zou GW (2009) Isolation and characterization of polymorphic microsatellite markers in the yellow catfish, Pelteobagrus fulvidraco. Conserv Genet Resour 1: 63.

Meyer-Sand BR, Blanc-Jolivet C, Mader M, Paredes-Villanueva K, Tysklind N, Sebbenn AM, Guichoux E, Degen B (2018) Development of a set of SNP markers for population genetics studies of Ipe (Handroanthus sp.), a valuable tree genus from Latin America. Conserv Genet Resour 10: 779-781.

Nelson JS, Grande TC, Wilson MV (2016) Fishes of the World. Hoboken: John Wiley \& Sons Press.

Rychlik W (2007) OLIGO 7 primer analysis software. Methods Mol Biol 402: 35-60.

Yeh FC, Yang R, Boyle TB, Ye Z, Mao JX (1997) POPGENE, the user-friendly shareware for population genetic analysis. Molecular Biology and Biotechnology Centre, University of Alberta, Canada 10: 295-301.

Yu JN, Kim SK, Sagong J, Ryu SH, Chae B (2019) Identification of microsatellite markers and their application in yellow catfish (Pseudobagrus fulvidraco Richardson, 1846) population genetics of Korea. J Genet 98: 2.

Zhang X, Li Y, Gao Z, Wang W (2009) Isolation and characterization of polymorphic microsatellite loci from yellow catfish (Pelteobagrus fulvidraco). Conserv Genet Resour 1: 313-315.

Zhou H, Duan G, Jiang H, Ling J, Hu Y, Zhang Y, Wang H, Pan T, Chen X (2021) High-density genetic map and QTL mapping for body color of the yellow catfish (Tachysurus fulvidraco $₫ \times$ T. vachellii ). Anim Genet 52: 246-248. 